\title{
Should we do myomectomy in women with infertility? A new criteria for decision making
}

\section{Oleksiy Aleksenko, Valentin Potapov, Mykhailo Medvediev SE "Dnipropetrovsk Medical Academy of Health Ministry of Ukraine" Department of Obstetrics and Gynecology}

* - difference is statistically significant, $p=0,01$
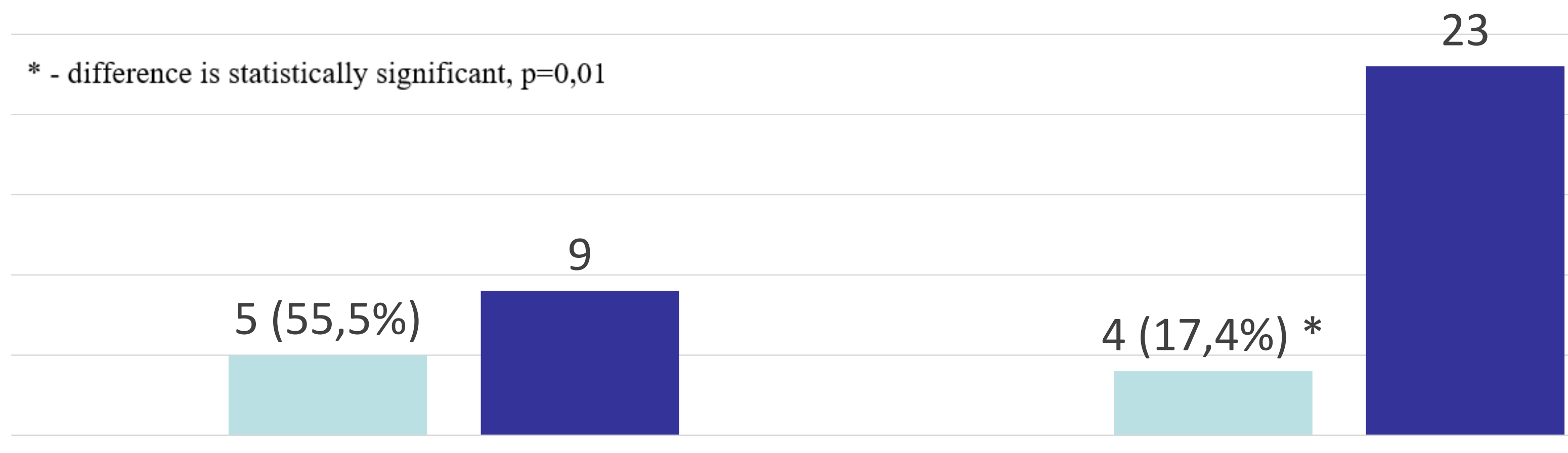

\section{Good prognosis peristalsis}

Poor prognosis peristalsis

\section{Pregnancy Infertility}

The role of uterine leiomyoma in women of reproductive age as a factor that influences fertility has been subject of debate for many years. It's importance as a research topic continues to grow as recently more women delay childbirth until later period of their life. It's well known that submucosal myomas (types 0-2) are clearly associated with infertility and especially pregnancy loss. In case of the presence of such myoma deforming uterine cavity in most of the cases a hysteroscopic myoma resection would be appropriate option before attempts of conception. Association of leiomyoma without deformity of uterine cavity (FIGO type 3 ) with poor pregnancy prognosis is not so obvious. In this connection, an objective prediction of the potential impact of asymptomatic uterine leiomyoma on fertility and pregnancy outcome is of special importance. Reliable prediction of negative myoma impact on future pregnancy course could help with clinical decision about necessity of surgical intervention in only patients with 'poor prognosis' avoiding invasive procedures in the rest of patients.

It was found that the uterine peristalsis (contractility) of non-pregnant uterus can influence female fertility. It is believed that one of the mechanisms of uterine leiomyoma negative impact on fertility can be a change of amplitude and direction of normal uterine contractility in periovulatory period and during the "window of implantation." There's some evidence that myomectomy could improve reproductive function and pregnancy outcomes in patients with abnormal patterns of uterine peristalsis.

In our study 32 reproductive age patients with type 3 myoma have been included. In all patients a computer-based analysis of uterine peristalsis has been performed during ovulation and 'implantation window'. Among this group of patients 23 women were with 'poor prognosis' pattern of contractions and 9 were with 'good prognosis' (fig.1). After one year of pregnancy planning 5 of 9 of ' $g o o d$ prognosis contractility' got pregnant $(55.5 \%)$ and only 4 of 23 'poor prognosis contractility' women $(17.4 \%)$ with statistically significant difference $(\mathrm{p}=0,01)$.
Furthermore, 14 of 19 patients in "poor prognosis" group decided to perform laparoscopic myomectomy after informed consent was signed. Surgery with standard technique was uneventful in all cases. 3 months post op the uterine contractility was reevaluated. It has been shown that after myomectomy uterine contractility had become "good prognosis" in 10 of 14 patients $(71,4 \%)$. Patients were allowed to try spontaneous conception after 6 months post surgery. During 1 year of observation 7 of 10 patients in "good prognosis" peristalsis group after myomectomy (70\%) got pregnant and only 1 of 4 patients with "poor prognosis" peristalsis after myomectomy $(25 \%)$.

Investigation of uterine contractility in women with uterine leiomyoma non-deforming uterine cavity (type 3) may be one of the criteria that can be used for decision about whether myomectomy should be performed avoiding unnecessary risks of surgery in women with 'good prognosis peristalsis'. Laparoscopic myomectomy probably can improve fertility in those patients who had infertility associated with "poo prognosis" uterine peristalsis. We need more data to clarify this hypothesis.

\section{Sources}

1. Meirzon D. A new method for analysis of non-pregnant uterine peristalsis using transvaginal ultrasound / D. Meirzon, A. J. Jaffa, Z. Gordon, D. Elad // Ultrasound Obstet. Gynecol. 2011. - Vol.38. - P.217-224.

2. Bulletti C. Uterine contractility during the menstrual cycle / C. Bulletti, de D. Ziegler, V. Polli et al. // Hum. Reprod. - 2000. - Vol.15, Suppl. 1. - P.81-89.

3. Aguilar H.N. Physiological pathways and molecular mechanisms regulating uterine contractility / H.N. Aguilar, B.F. Mitchell // Human Reproduction Update. - 2010. - Vol.16, N.6. - P. 725-744, 2010 «Physiological pathways and molecular mechanisms regulating uterine contractility»

4. Fanchin R. Uterine dynamics: impact on the human reproduction process / R. Fanchin, J.M. Ayoubi // Reprod Biomed Online. - 2009. Vol.18, Suppl 2. - P.57-62. 